Research Article

\title{
Sequence characterization, in silico mapping and cytosine methylation analysis of markers linked to apospory in Paspalum notatum
}

\author{
Maricel Podio ${ }^{1,2}$, María P. Rodríguez ${ }^{1}$, Silvina Felitti ${ }^{1}$, Juliana Stein ${ }^{1}$, Eric J. Martínez ${ }^{2}$, Lorena A. Siena ${ }^{1}$, \\ Camilo L. Quarin ${ }^{2}$, Silvina C. Pessino ${ }^{1}$ and Juan Pablo A. Ortiz ${ }^{1,2}$ \\ ${ }^{1}$ Laboratorio de Biología Molecular, Facultad de Ciencias Agrarias, Universidad Nacional de Rosario, \\ Zavalla, Santa Fe, Argentina. \\ ${ }^{2}$ Instituto de Botánica del Nordeste, Facultad de Ciencias Agrarias, Universidad Nacional del Nordeste, \\ Corrientes, Argentina.
}

\begin{abstract}
In previous studies we reported the identification of several AFLP, RAPD and RFLP molecular markers linked to apospory in Paspalum notatum. The objective of this work was to sequence these markers, obtain their flanking regions by chromosome walking and perform an in silico mapping analysis in rice and maize. The methylation status of two apospory-related sequences was also assessed using methylation-sensitive RFLP experiments. Fourteen molecular markers were analyzed and several protein-coding sequences were identified. Copy number estimates and RFLP linkage analysis showed that the sequence PnMA/3 displayed 2-4 copies per genome and linkage to apospory. Extension of this marker by chromosome walking revealed an additional protein-coding sequence mapping in silico in the apospory-syntenic regions of rice and maize. Approximately $5 \mathrm{~kb}$ corresponding to different markers were characterized through the global sequencing procedure. A more refined analysis based on sequence information indicated synteny with segments of chromosomes 2 and 12 of rice and chromosomes 3 and 5 of maize. Two loci associated with apomixis locus were tested in methylation-sensitive RFLP experiments using genomic DNA extracted from leaves. Although both target sequences were methylated no methylation polymorphisms associated with the mode of reproduction were detected.
\end{abstract}

Keywords: apomixis, chromosome walking, gene mapping, molecular markers.

Received: February 27, 2012; Accepted: July 1, 2012.

\section{Introduction}

Apomixis is a route of asexual reproduction through seeds (Nogler, 1984). This mode of reproduction has been described in about 400 genera from 40 plant families and seems to have arisen multiple times during evolution (Carman, 1997). Apomixis avoids meiosis and fertilization of the egg cell to generate progeny that are clones of the mother plant (Savidan, 2000). In gametophytic apomixis, the type of apomixis found in the Poaceae, embryo sacs bearing non-reduced nuclei are formed from the megaspore mother cell itself (diplospory) or from nucellar cells (apospory), followed by development of the embryo through parthenogenesis from unreduced ( $2 n$ ) egg cells. Depending on the species, the endosperm develops after fertilization of the polar nuclei (pseudogamy) or autonomously (Koltunow, 1993). Gametophytic apomixis tends to occur in poly-

Send correspondence to Juan Pablo A. Ortiz. Laboratorio de Biología Molecular, Facultad de Ciencias Agrarias, Universidad Nacional de Rosario, Campo Experimental Villarino s/n, CC 14 (S2125 ZAA), Zavalla, Santa Fe, Argentina. E-mail: jortiz@unr.edu.ar; jpaortiz@yahoo.com.ar. ploids, most often at the tetraploid stage or higher levels (Asker and Jerling, 1992). Despite the widespread occurrence of apomixis in angiosperms, the trait is rare in crop gene pools, although apomictic wild relatives have been identified for important cereals, including maize and wheat (Spillane et al., 2004).

Several studies have examined the inheritance of apomixis in tropical and subtropical forage grasses (reviewed by Ozias-Akins and van Dijk, 2007). These species are usually polyploid, highly heterozygous and genetically poorlycharacterized. Transferring apomixis to sexually reproducing crops could have an enormous impact in agriculture. The most important potential benefits derived from harnessing apomixis would be the fixation and cloning of elite genotypes and hybrids through seeds, as well as the multiplication of vegetatively-reproducing crops via seeds (Vielle-Calzada et al., 1996; Savidan, 2000; Toenniessen, 2001).

Paspalum notatum Flügge (bahiagrass) is a polymorphic forage grass native to South America. Tetraploid cytotypes (common bahiagrass) are important natural forage resources in tropical and subtropical areas of southern 
Brazil, Paraguay and northeastern Argentina; their reproduction is by nearly-obligate aposporous apomixis (Gates et al., 2004). Completely sexual tetraploid plants have never been collected from nature, although several individuals have been artificially created by colchicine treatment of diploids or by crossing highly sexual facultative tetraploid genotypes (Quarin et al., 2003). Experimentallygenerated tetraploid sexual plants have allowed the development of intraspecific populations that segregate according to their mode of reproduction; these populations have been used for genetic and molecular studies of apomixis in the species.

Inheritance analyses done on $\mathrm{F}_{1}, \mathrm{~F}_{2}$ and $\mathrm{BC}_{1}$ families derived from sexual $\mathrm{x}$ apomictic crosses indicated that apospory in tetraploid $P$. notatum is controlled by a single locus with a distorted segregation ratio, probably due to a pleiotropic lethal effect with incomplete penetrance of the allele controlling apospory or, alternatively, to a partially lethal linked factor (Martínez et al., 2001). A group of RAPD, RFLP and AFLP molecular markers linked to the apospory locus has been identified (Martínez et al., 2003; Pupilli et al., 2004; Stein et al., 2004, 2007; Rebozzio et al., 2012). These markers define a chromosome block characterized by recombination restriction and preferential chromosome pairing (Martínez et al., 2003; Stein et al., 2004). Several AFLP and two SCAR markers (SPNA1 and $S P N A 2$ ) linked to the trait are consistently present in a group of apomictic accessions from diverse geographic origins (Rebozzio et al., 2012). These findings suggest that the structure of the chromosome segment carrying apospory is highly conserved in apomictic races of this species.

Based on the mapping data reported by Stein et al. (2007), the apospory region of tetraploid P. notatum may consist of a large chromosomal segment. A comparative analysis done with RFLP markers previously mapped in rice showed that clones $\mathrm{C} 560$ and $\mathrm{C} 932$ (rice chromosomes 2) and C545, C996A and C1069 (rice chromosome 12) mapped completely linked to apospory in this species (Pupilli et al., 2004). Synteny between the apospory locus and rice chromosome 12 was conserved in at least other three Paspalum species (P. mallacophyllum, P. simplex and P. procurrens) (Pupilli et al., 2004; Hojsgaard et al., 2011). Information derived from comparative mapping analyses in apomixis research is extremely valuable since this trait is currently considered to represent a reproductive deviation caused by mutations and/or epimutations involving canonical sexual pathway genes, rather than the development of a new function. Consequently, gene order comparisons with sexual model grass species for which the genomes have been fully sequenced could allow rapid, exhaustive identification of candidate genes whose structure and/or expression may be affected in polyploid, highly heterozygous and poorly characterized apomictic species.

Although the structure of the chromosomal segment carrying apospory in $P$. notatum has been partially charac- terized by using molecular markers (Martínez et al., 2003; Pupilli et al., 2004; Stein et al., 2007) there is currently no sequence information for this region. The sequencing of markers fully linked to apospory would allow a detailed analysis of synteny involving model grasses such as rice and maize, and facilitate the identification of candidate genes physically associated with apospory. We are aware that characterizing the locus that controls apospory in $P$. notatum is a difficult task since polyploidy, heterozygosity, a lack of recombination and the presence of retrotransposons can complicate strategies used to recover specific sequence and provide unequivocal positional validation. However, since no sequence information is available for this species, data derived from markers linked to apospory would help to refine the study of synteny and accelerate the identification of candidate genes related to apospory.

The objective of this work was to analyze the sequences of molecular markers linked to apospory in $P$. notatum, extend them by chromosome walking and perform an in silico analysis of synteny in maize and rice. The cytosine methylation of markers linked to apospory were assessed by methylation-sensitive RFLP.

\section{Materials and Methods}

\section{Plant material}

The plant material used in this work consisted of the tetraploid $(2 \mathrm{n}=4 \mathrm{x}=40)$ genotypes Q4188 and Q4117 and an $\mathrm{F}_{1}$ mapping population derived from them. Q4188 is a completely sexual experimental hybrid derived from a cross between a highly sexual genotype (Q3664) and a natural apomictic plant (Quarin et al., 2003) and Q4117 is an obligate apomictic tetraploid accession collected from southern Brazil (Ortiz et al., 1997). Along with the parental genotypes, $65 \mathrm{~F}_{1}$ individuals (55 sexual and 10 aposporous) were used to corroborate the linkage between molecular markers and apospory. The $\mathrm{F}_{1}$ hybrids were the remaining part of a larger mapping population of 113 individuals that segregated according to the mode of reproduction developed by Stein et al. (2004). This population was used to identify molecular markers linked to apospory, to determine the type of inheritance in tetraploid races and to construct a genetic linkage map of the species (Stein et al., 2004, 2007). All of the hybrids were initially classified according to their mode of reproduction based on cytoembryological observations and molecular analysis (Stein et al., 2004).

\section{Molecular markers linked to apospory}

Two RAPD markers (BCU243-377 and BCU2591157) (Martínez et al., 2003), 14 AFLP markers (E32M33e, E33M32c, E33M33a, E33M33b, E33M33f, E33M42e, E33M42g, E35M33p, E35M33n, E36M37c, E36M37d, E36M38a, E35M43n and E35M43p) (Stein et al., 2004, 2007) and five cDNA RFLP clones (C560, C932, 
C454, C996A and C1069, from the New Landmarker set, Rice Genome Research Program, Japan), previously reported as completely linked to apospory in tetraploid $P$. notatum (Martínez et al., 2003; Pupilli et al., 2004) were analyzed. The RAPD and AFLP markers were re-amplified as described by Martínez et al. (2003) and Stein et al. (2007), respectively, from genomic DNA of Q4188, Q4117 and all $\mathrm{F}_{1}$ progenies. Markers linked to apospory were identified on agarose or polyacrylamide gels based on their molecular weight and cosegregation with the mode of reproduction. Target bands from Q4117 were excised from the gels and eluted in buffer containing $0.5 \mathrm{M}$ ammonium acetate and $1 \mathrm{mM}$ EDTA, $\mathrm{pH}$ 8. DNA fragments were precipitated with absolute ethanol, dried at room temperature, dissolved in $20 \mu \mathrm{L}$ of distilled water, re-amplified using the corresponding RAPD or AFLP primers and purified with the DNA Wizard SV Gel and PCR Clean-up system (Promega). Clean fragments were cloned with the pGEM-T Easy Vector system (Promega). Inserts of recombinant plasmids were sequenced by Macrogen Inc. (Korea). The sequences of RFLP clones were retrieved from the GRAMENE web page (www.gramene.org).

\section{Chromosome walking procedure}

Genomic sequences flanking the AFLP-derived fragment PnMAI3 were recovered from genomic DNA of Q4117 by using the Genome Walker ${ }^{\mathrm{TM}}$ universal kit protocol (Clontech Laboratories, Inc.). The outer adaptor primer AP1 (see GenomeWalker universal kit user manual) and an outer sequence-specific primer were used (Table S1) for the first amplification. The PCR products were used as templates for a second PCR with the nested adaptor primer AP2 (GenomeWalker) in combination with nested sequencespecific primers (Table S1). The PCR products were electrophoresed on $2 \%$ agarose gels and the bands of interest were cut out, cloned and sequenced as described above. The extended fragments were validated by searching for the corresponding upper and lower primers and aligning the overlapping segments with the original PnMAI3 sequence. Alignments were done with Sequencher 4.10.1 Demo Version (Gene Codes Corporation). Contigs between PnMAI3 and the flanking sequences were assembled with MegAlign v.4.03 (DNASTAR Inc.).

\section{Amplification of apospory-specific contigs from genomic DNA}

Apospory-specific contigs were amplified from Q4188 and Q4117 genomic DNA by PCR using internal primers designed within each extended sequence in combination with anchored primers aligned with PnMAI3 (Table S2). The PCR mixtures used $100 \mathrm{ng}$ of DNA, $30 \mathrm{ng}$ of forward and reverse primers, $1 \mathrm{X} \mathrm{Taq}$ buffer (Promega), $200 \mathrm{mM}$ of each dNTP, $1 \mathrm{mM} \mathrm{MgCl} 2$ and $1 \mathrm{U}$ of Taq polymerase (Promega). The amplification reactions included an initial step of 2 min at $94{ }^{\circ} \mathrm{C}$ followed by seven cycles of
1 min at $94{ }^{\circ} \mathrm{C}, 1$ min at $62{ }^{\circ} \mathrm{C}$ and 1 min at $72{ }^{\circ} \mathrm{C}$ with decreases in the annealing temperature of $1{ }^{\circ} \mathrm{C}$ per cycle, followed by 35 cycles of $1 \mathrm{~min}$ at $94^{\circ} \mathrm{C}, 1 \mathrm{~min}$ at $55^{\circ} \mathrm{C}$ and $1 \mathrm{~min}$ at $72{ }^{\circ} \mathrm{C}$ with a final incubation at $72{ }^{\circ} \mathrm{C}$ for $5 \mathrm{~min}$. The PCR products were separated by electrophoresis on $6 \%$ polyacrylamide gels at $60 \mathrm{~W}$ for $1 \mathrm{~h}$ and silver-stained. The linkage between amplicons and apospory was tested in 10 sexual and 10 aposporous $F_{1}$ individuals of the mapping population.

\section{In silico mapping analysis of Paspalum notatum apospory-specific sequences}

Sequences of molecular markers linked to apospory in $P$. notatum were located in the rice and maize genomes by using the BLAST (Altschul et al., 1990) tool through the GRAMENE and MaizeSequence web pages, respectively. Putative orthologous sequences were determined based on the procedure described by Salse et al. (2004) by applying the criterion of at least $65 \%$ identity over at least $60 \%$ of the length of the sequences at E-values $<0.005$.

\section{RFLP analyses of Paspalum notatum apospory-specific sequences}

Genomic DNA was extracted from leaf tissue as described by Martínez et al. (2003). Three clones (PnMAC5, $P n M A I 3$ and $P n A M 3)$ derived from AFLP markers linked to apospory were used as RFLP probes. Hybridizations were done using a non-radioactive procedure (Ortiz et al., 2001) on Nylon Hybond N membranes (Amersham-Pharmacia) containing $20 \mu \mathrm{g}$ of DNA alternatively digested with three restriction enzymes (EcoRI, HindIII and BamHI or PstI). Putative genetic linkage between markers and apospory was assessed by bulk segregant analysis (BSA) (Michelmore et al., 1991) in which 10 sexual (BS) and 10 aposporous (BA) $F_{1}$ progenies were used to construct each group.

\section{Methylation-sensitive RFLP analysis of markers linked to apospory}

Methylation-sensitive RFLP experiments were done using the isoschizomers MspI and HpaII in combination with the apospory-specific clones PnMAM3 and C1069. MspI and HpaII recognize the same target sequence (CCGG) but exhibit different sensitivities to the cytosine methylation status (McClelland et al., 1994; Tardy-Planechaud et al., 1997). Genomic DNA was extracted as described above from leaf tissue of plants grown in a greenhouse. The methylation conditions of the target sequences were determined by comparing the hybridization patterns generated by the isoschizomers from a given sample. Linkage between markers and the mode of reproduction was estimated by BSA analysis and the corresponding de-bulk assay that included 10 sexual and 10 aposporous $F_{1}$ progenies, as described by Martínez et al. (2003). 


\section{Results}

Sequence analysis of molecular markers completely linked to apospory

Two RAPD and 14 AFLP molecular markers previously reported to be completely linked to apospory in tetraploid $P$. notatum were re-amplified using the corresponding primers from genotypes Q4188 (sexual), Q4117 (aposporous) and their $\mathrm{F}_{1}$ hybrids (55 sexual and 10 aposporous). The amplification products were electrophoresed and markers linked to apospory were identified based on their molecular weight and strict cosegregation with the mode of reproduction of the $\mathrm{F}_{1}$ plants. The bands of interest were isolated from Q4117, cloned and sequenced. Of the 16 markers tested (two RAPDs and 14 AFLPs), only nine (one RAPD and eight AFLPs) were successfully recovered and cloned (Table 1). The rest of the PCR-based markers were lost during the cloning step or could not be confirmed because they lacked specific primers. A consensus was built for each marker by assembling sequences from at least three clones of the same fragment, with a minimum homology of $95 \%$. The sequences of the cloned fragments ranged in length from $42 \mathrm{bp}$ to $351 \mathrm{bp}$ (Table 1). BLAST analysis revealed that only three of the sequences $(33.3 \%$ of the total sequences characterized) shared significant homology with sequences in the databases; the others probably represented poorly-conserved intergenic sequences. Fragment PnMA243 was similar to a cDNA clone of Panicum virgatum and sequence PnMAJ5 aligned with a P. virgatum genomic clone. Sequence PnMAM3 shared similarity with rice locus LOC_Os07g22800.1 that encodes a putative Ty1-copia subclass retrotransposon protein (Table 1).

RFLP markers from rice cDNA clones that mapped at the $P$. notatum apospory locus encoded for L6 and L14 ribosomal proteins (C560 and C996, respectively), a peptidyl-prolyl cis-trans isomerase protein (C932), a $\mathrm{KH}$ domain-containing protein $(\mathrm{C} 454)$ and a mutator subclass transposon protein (C1069) (Table 1).

\section{Copy number estimation of apospory-associated sequences}

The AFLP-derived clones PnMAC5, PnMAI3 and PnMAM3 were used as probes in Southern blot experiments with genomic DNA from the Q4188 and Q4117 genotypes in order to determine the copy number of these clones in the P. notatum genome. Clone PnMAM3 was included as a high-copy number control since it showed similarity with retrotransposons in the BLAST searches (Table 1). Hybridization with the control sequence (PnMAM3) resulted in a smeared pattern with some discrete bands, a

Table 1 - Sequences identified by RAPD, AFLP and RFLP molecular markers linked to apospory in P. notatum.

\begin{tabular}{|c|c|c|c|c|c|}
\hline Marker type & $\begin{array}{l}\text { Marker linked to } \\
\text { apospory }\end{array}$ & $\begin{array}{l}\text { Sequence iden- } \\
\text { tity }\end{array}$ & Length (bp) & Expected value & Best Blastn/Blastx alignment $^{1}$ \\
\hline RAPD & $\mathrm{BCU} 243-377^{\mathrm{a}}$ & PnMA243 & 351 & $5.0 \mathrm{e}^{-50}$ & $\begin{array}{l}\text { gb FL982880.1 Panicum virgatum cDNA clone. Sim- } \\
\text { ilar to hypothetical protein DNA binding protein }\end{array}$ \\
\hline \multirow[t]{8}{*}{ AFLP } & $\mathrm{E} 32 \mathrm{M} 33 \mathrm{e}^{\mathrm{b}}$ & PnMAC5 & 96 & - & n.s. \\
\hline & $\mathrm{E} 33 \mathrm{M} 32 \mathrm{c}^{\mathrm{b}}$ & PnMAI3 & 279 & - & n.s. \\
\hline & $\mathrm{E} 33 \mathrm{M} 42 \mathrm{e}^{\mathrm{b}}$ & PnMAJ5 & 207 & $2.0 \mathrm{e}^{-07}$ & $\begin{array}{l}\text { gb AC243221.1 Panicum virgatum clone } \\
\text { PV_ABa006-D05 }\end{array}$ \\
\hline & $\mathrm{E} 33 \mathrm{M} 42 \mathrm{~g}^{\mathrm{b}}$ & PnMAJ7 & 115 & - & n.s. \\
\hline & E36M37 $c^{b}$ & PnMAM3 & 87 & $7.3 \mathrm{e}^{-06}$ & $\begin{array}{l}\text { Os } 07 \mathrm{~g} 22800.1 \text { retrotransposon protein putative } \\
\text { Ty1-copia subclass }\end{array}$ \\
\hline & E36M38 $\mathrm{a}^{\mathrm{b}}$ & PnMAN1 & 42 & & n.s. \\
\hline & E35M43n ${ }^{b}$ & PnMAU14 & 91 & & n.s. \\
\hline & $\mathrm{E} 35 \mathrm{M} 43 \mathrm{p}^{\mathrm{b}}$ & PnMAU16 & 176 & & n.s. \\
\hline \multirow[t]{5}{*}{ RFLP } & $\mathrm{C} 560^{\mathrm{c}}$ & $D 15383$ & 421 & $2.7 \mathrm{e}^{-53}$ & $\begin{array}{l}\text { LOC_Os04g3970060S ribosomal protein L6 puta- } \\
\text { tive expressed }\end{array}$ \\
\hline & $\mathrm{C} 932^{\mathrm{c}}$ & D22694 & 329 & $5.0 \mathrm{e}^{-152}$ & $\begin{array}{l}\text { LOC_Os02g52290.1 peptidyl-prolyl cis-trans } \\
\text { isomerase FKBP-type }\end{array}$ \\
\hline & $\mathrm{C} 454^{\mathrm{c}}$ & C98049 & 725 & $2.7 \mathrm{e}^{-113}$ & LOC_Os12g40560.1 KH domain-containing protein \\
\hline & C996A ${ }^{c}$ & C98189 & 271 & $1.2 \mathrm{e}^{-49}$ & LOC_Os12g42180 50S ribosomal protein L14 \\
\hline & $\mathrm{C} 1069^{\mathrm{abc}}$ & D15675 & 400 & $1.8 \mathrm{e}^{-228}$ & $\begin{array}{l}\text { LOC_Os } 12 \mathrm{~g} 40530.1 \text { transposon protein putative } \\
\text { mutator sub-class }\end{array}$ \\
\hline
\end{tabular}

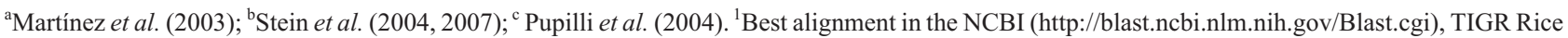
Genome Annotation (http://blast.jcvi.org/euk-blast/index.cgi?project=osa1), GRAMENE (http://www.gramene.org/) and MaizeSequence database (http://www.maizesequence.org/index.html). n.s. = no significant similarity found. GenBank accession numbers for nucleotide sequences: $P n M A 243=$ $\mathrm{JN} 250998$, PnMAI3 $=\mathrm{JN} 250999$ and PnMAJ5 $=\mathrm{JN} 251000$. 
characteristic of highly repetitive sequences (Table 1, Figure 1A). Assays with PnMAC5 showed only one hybridization band with the three restriction enzymes tested and no polymorphisms between parental plants (Figure 1B). Clone PnMAI3 showed 2-4 hybridization bands with polymorphic fragments between Q4188 and Q4117 for two restriction enzymes (Figure 1C). The polymorphism revealed by $E c o R I$ consisted of bands with different migration rates in Q4188 and Q4117, while the polymorphism produced by Pst I consisted of a band present in Q4117 and absent in Q4188 (Figure 1C). To determine the association between polymorphisms and the mode of reproduction, bulked segregant analysis (BSA) was done by hybridizing PnMAI3 on both sexual (BS) and apomictic (BA) bulks (Figure 1D). The hybridization pattern showed that the polymorphisms detected between parental plants were also observed between sexual and apomictic bulks (Figure 1C and D), indicating genetic linkage between the target sequence and apospory.

\section{Characterization of PnMAI3-flanking sequences by chromosome walking}

Based on results described above, we initiated chromosome walking around PnMAI3 since this clone had a low-copy number and showed linkage to apospory. Successive rounds of amplification yielded four consensus sequences (\%ID > 80), two of them extending towards the 5 flanking region and two towards the 3 flanking region, respectively (Table 2). Only fragments that could be validated by identifying the primers used during the second round of amplification and by assembling the corresponding contig with the original sequence were considered for analysis. The length of the extended fragments ranged from $54 \mathrm{bp}$ to $133 \mathrm{bp}$ for the 5' flanks and $102-826$ bp for the 3 ' flanks, respectively. These results agreed with the copy number (2-4) estimated for this marker, indicating that the

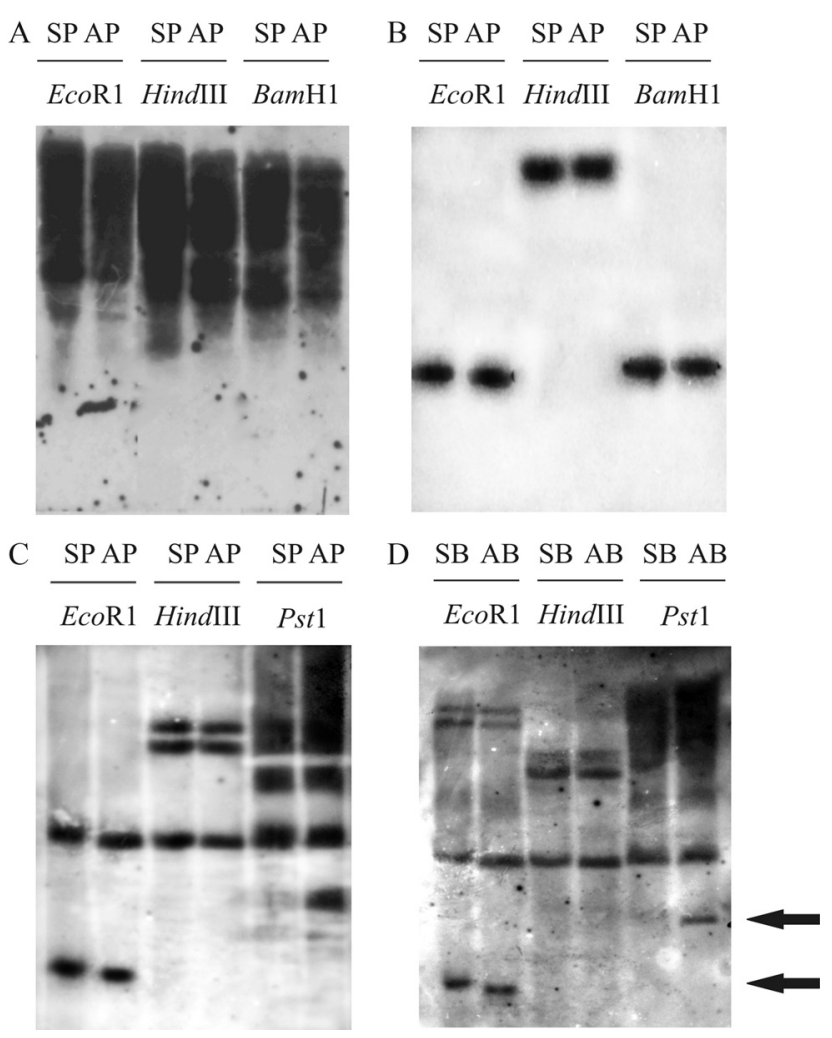

Figure 1 - Southern blot of apospory-specific AFLP-derived sequences in DNA from sexual and apomictic Paspalum notatum. A, B and C: Hybridization of DNA from genotypes Q4188 (SP) and Q4117 (AP) with clones PnMAM3, PnMAC5 and PnMAI3, respectively, after digestion with three restriction enzymes. D: Hybridization of PnMAI3 against sexual (SB) and apomictic $(\mathrm{AB})$ bulks obtained from ten $\mathrm{F}_{1}$ sexual and apomictic progenies, respectively. Arrows indicate polymorphic bands between parental plants and sexual and apomictic bulks.

Table 2 - Characterization of $P$. notatum genomic contigs derived from extension of the apospory-specific marker PnMAI3.

\begin{tabular}{|c|c|c|c|c|c|}
\hline $\begin{array}{l}\text { Extension } \\
\text { direction }\end{array}$ & Contig name & Length (bp) & E value & Best alignment (Blastn) /Annotation ${ }^{1}$ & $\begin{array}{l}\text { In silico mapping onto rice and maize } \\
\text { genomes }{ }^{1}\end{array}$ \\
\hline \multirow[t]{2}{*}{5 , } & PnGSA1 & 350 & $3 e^{-08}$ & $\begin{array}{l}\text { gbFE614154.1 CBYX11857.b1 CBYX } \\
\text { Panicum virgatum callus cDNA }\end{array}$ & No significant hits in the rice genome \\
\hline & $\operatorname{Pn} G S A 2$ & 384 & $2 \mathrm{e}^{-05}$ & $\begin{array}{l}\text { gbJG806067.1 CFNU4264.b1 CFNU } \\
\text { Panicum virgatum shoot cDNA }\end{array}$ & No significant hits in the rice genome \\
\hline \multirow[t]{4}{*}{$3^{\prime}$} & $P n G S A 3$ & 689 & $2 \mathrm{e}^{-59}$ & $\begin{array}{l}\text { gbCD438587.1 Endosperm_5 Zea mays } \\
\text { cDNA LOC100281493 } \\
\text { N6-adenosine-methyltransferase } \\
\text { MT-A70-like protein }\end{array}$ & $\begin{array}{l}\text { OS 2. Position: } 27358073-27358338 \\
\left(\mathrm{E}-\mathrm{val}: 5.1 \mathrm{e}^{-42}\right) . \text { In the proximity of sev- } \\
\text { eral apospory-linked markers }\end{array}$ \\
\hline & & & $5.1 e^{42}$ & $\begin{array}{l}\text { LOC_Os02g45110.1 Transcript. } \\
\text { MT-A70 domain containing protein }\end{array}$ & $\begin{array}{l}\text { ZM 5. Position: } 194339512 \text { - } \\
194343577 \text { (E-val: } 4.2 \mathrm{e}^{-28} \text { ). In the prox- } \\
\text { imity of several apospory-linked markers }\end{array}$ \\
\hline & $\operatorname{PnGSA4}$ & 1077 & $8 \mathrm{e}-{ }^{54}$ & $\begin{array}{l}\text { gb CD433356.1 EL01N0307F02.b Endo- } \\
\text { sperm_3 Zea mays cDNA }\end{array}$ & $\begin{array}{l}\text { Multiple alignments (lowest E-val: } 1.9 \\
\mathrm{e}^{-25} \text { ) }\end{array}$ \\
\hline & & & $1.9 \mathrm{e}^{25}$ & $\begin{array}{l}\text { LOC_Os } 03 \mathrm{~g} 45030.1 \text { Retrotransposon } \\
\text { protein putative Ty3-gypsy subclass }\end{array}$ & \\
\hline
\end{tabular}

${ }^{1}$ Determined by BLAST analysis (Altschul et al., 1990) via the GRAMENE and MaizeSequence web pages. OS: rice chromosome, ZM: maize chromosome. 
fragment was located in at least two loci, probably surrounded by different flanking sequences. The sequences of contigs PnGSA1 and PnGSA2 (5' amplifications) were similar to cDNA from $P$. virgatum calli and shoots, respectively, while the sequence of $P n G S A 3$ was highly similar to maize endosperm cDNA (gb CD438587.1) that encodes a N6-adenosine-methyltransferase MT-A70-like protein. The PnGSA3 sequence also shared high similarity with LOC_Os02g45110.1 that encodes an MT-A70 domaincontaining protein of rice. Contig PnGSA4 was similar to a maize EST expressed in endosperm and to locus LOC_Os03g45030.1 of rice that encodes a putative retrotransposon protein (Table 2).

PCR amplifications based on primers that covered the four extended sequences and the original marker (Table S2) were used to amplify the whole contig in order to verify the presence of contigs derived from extension of the apospory-specific sequence PnMAI3 in the P. notatum genome (thereby excluding possible contamination or chimeric assemblies) and to determine which of the two sequences isolated at each flank mapped to the apospory locus. Amplifications done on genomic DNA from both parental plants showed fragments of the expected size, confirming correct assembly, in addition to other fragments. However, the expected bands showed no polymorphisms between parental genotypes. This outcome prevented mapping the fragments using the $\mathrm{F}_{1}$ hybrids. To overcome this difficulty, we undertook an in silico mapping analysis with the contig sequence on rice to distinguish which one was located in the region previously associated with apospory reported by Pupilli et al. (2004). Table 2 summarizes the in silico mapping results. The two 5'-flanking regions showed no significant match with the rice genome in GRAMENE BLAST searches, even though they showed homology to two Panicum virgatum cDNAs. These sequences may represent novel sequences present only in a group of related grasses. Of the two 3'-flanking regions, PnGSA3 mapped to rice chromosome 2, close to RFLP probes previously associated with apomixis (see below). The other 3'-flanking region (PnGSA4) showed multiple alignments and probably represented a copy of a repetitive sequence located adjacent to a second PnMAI3 fragment but at a different genomic location.

Interestingly, during contig validation, several other fragments of unexpected size were amplified. Some of these were polymorphic between Q4188 and Q4117 (Figure S1). Additional linkage analyses to determine the association between polymorphic bands and the mode of reproduction were done using the corresponding primers for each contig on DNA from both parental plants and a sample of 20 ( 10 sexual and 10 aposporous) $\mathrm{F}_{1}$ individuals. PCR amplifications showed that most fragments were unlinked to apospory. However, amplification of sequence PnGSA1 with primers VI3R and VI35BF1 generated a band of 764 bp (PnMA764) that was present in Q4117 and in all aposporous individuals but was absent in Q4188 and in all sexual $F_{1}$ progenies (Figure $S 2$ ). This fragment (GenBank accession number: JN25001) showed sequence similarity to a maize transposable element (GRMZM5G800837_T01). Likewise, amplification of PnGSA4 with primers VI3F and VI3310AR2 showed a high molecular weight band (PnMA1200) that also cosegregated with apospory (not shown). However, all attempts to clone this marker failed and their sequence could not be analyzed.

\section{In silico mapping analysis of the whole set of apospory-associated sequences}

All sequences derived from the $P$. notatum apospory-specific markers were mapped in silico onto the rice and maize genomes in order to determine the location of orthologous sequences in both model species. Rice RFLP clones previously associated with apospory were also included as reference points (Table 3). Putative orthologues to PnMA243 and PnMAM3 were found located on rice chromosome 2, close to the apospory-related markers C560 and C932. PnMA764 aligned with a genomic sequence of rice chromosome 12 and maize chromosome 5, near to the apospory-related rice clones $\mathrm{C} 1069$ and C932, respectively. Apospory-related sequences mapping onto rice chromosomes 2 and 12 and maize chromosomes 3 and 5 covered approximately $10 \mathrm{Mbp}$ and $30 \mathrm{Mbp}$, respectively (Figure 2). The rest of the sequences analyzed mapped on maize chromosomes 1 (C454), 4 (PnMA243, PnMAJ5 and C560) and 6 (PnMAJ5) (Table 3).

\section{Methylation-sensitive RFLP analysis of the apospory-related sequences}

Since the activity of repetitive elements is known to be controlled through specific cytosine methylation pat-

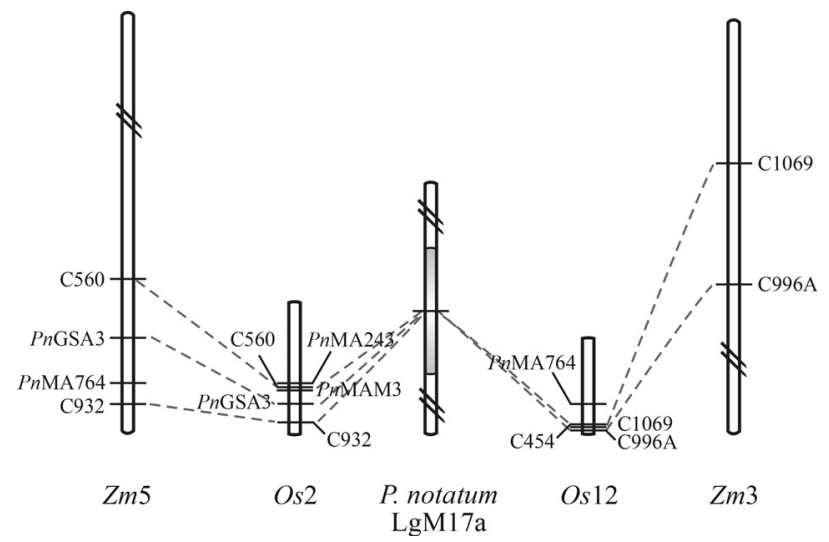

Figure 2 - In silico mapping of apospory-specific sequences in the rice and maize genomes. Paspalum notatum and rice sequences were located on rice $(O s)$ and maize $(\mathrm{Zm})$ chromosomes by using the BLASTn tool available at GRAMENE. The relative position of each marker was determined based on the physical position of the orthologous sequences listed in Table 3. Rice RFLP clones from rice chromosomes 2 and 12 that generated markers completely linked to apospory in the species were included as reference points. 
Table 3 - In silico mapping of $P$. notatum apospory-specific sequences in rice and maize genomes.

\begin{tabular}{|c|c|c|c|c|c|c|}
\hline Sequence & Rice chromosome (Os): $\mathrm{bp}^{1}$ & E value $^{2}$ & $\%$ ID & Maize chromosome (Zm): bp ${ }^{1}$ & E value $^{2}$ & $\% \mathrm{ID}$ \\
\hline PnMA243 & Os 2: 22235716 - 22235909 & 0.003 & 65.2 & Zm 4: 94738078 - 94738380 & $1.2 \mathrm{e}^{-11}$ & 65.9 \\
\hline \multirow[t]{2}{*}{ PnMAJ5 } & n.s. & n.s & n.s & $Z m$ 4: $181677342-181677525$ & $5.6 \mathrm{e}^{-07}$ & 70.3 \\
\hline & & & & Zm 6: $14579479-14579638$ & $6.6 \mathrm{e}^{-09}$ & 71.1 \\
\hline PnMAM3 & Os : multiple alignments & $7.6 \mathrm{e}^{-05}$ & 86.6 & $\mathrm{Zm}$ : multiple alignments & $7.2 \mathrm{e}^{-05}$ & 84.4 \\
\hline PnMA764 & Os 12: $18536262-18536706$ & $4.8 \mathrm{e}^{-7}$ & 68.2 & $\mathrm{Zm}:$ multiple alignments & $4.0 \mathrm{e}^{-05}$ & 67.6 \\
\hline \multirow[t]{2}{*}{ C560 } & Os 2: $22876845-22878075$ & $2.3 \mathrm{e}^{-147}$ & 100.0 & Zm 4: $128263137-128264614$ & $2.5 \mathrm{e}^{-79}$ & 86.9 \\
\hline & Os 4: 23476213 - 23477653 & $3.7 \mathrm{e}^{-213}$ & & Zm 5: 179392786 - 179393814 & $2.9 \mathrm{e}^{-79}$ & 87.2 \\
\hline C932 & Os 2: $32011864-32014026$ & $5 \mathrm{e}^{-152}$ & 100.0 & Zm 5: $209262954-209264371$ & $1.3 \mathrm{e}^{-53}$ & 87.0 \\
\hline C454 & Os 12: $25058216-25065293$ & 0.0 & 100.0 & $Z m$ 1: $167869383-167870468$ & $4.2 \mathrm{e}^{-98}$ & 75.1 \\
\hline C996A & Os 12: $26106507-26108035$ & $4.4 \mathrm{e}^{-160}$ & 100.0 & Zm 3: 86923502 - 86928449 & $1.0 \mathrm{e}^{-32}$ & 78.1 \\
\hline \multirow[t]{2}{*}{ C1069 } & Os 12: $25047073-25047471$ & $1.8 \mathrm{e}^{-228}$ & 100.0 & $Z m 1: 167565640-167566038$ & $1.0 \mathrm{e}^{-8}$ & 86.0 \\
\hline & & & & Zm 3: $115792597-115792988$ & $1.4 \mathrm{e}^{-75}$ & 85.5 \\
\hline$P n G S A 3$ & Os 2: $27358073-27358338$ & $5.1 \mathrm{e}^{-42}$ & 69.8 & Zm 5: 194339512 - 194343577 & $4.2 \mathrm{e}^{-28}$ & 79.6 \\
\hline PnGSA4 & Os: multiple alignments & $1.9 \mathrm{e}^{-25}$ & 66.3 & $Z m:$ multiple alignments & $8 \mathrm{e}^{-54}$ & 68.0 \\
\hline
\end{tabular}

${ }^{1}$ Orthologous sequences were assigned based on the criteria of $65 \%$ identity over at least $60 \%$ of the length of the sequences at E-values $<0.005$. This assignment was done using the Blastn tool available from GRAMENE and MaizeSequence. ${ }^{2}$ In cases of multiple alignments, the lowest E-value and the highest \%ID are indicated (corresponding to the best match).

terns, methylation-sensitive RFLP experiments were done by using the apospory-associated clones PnMAM3 (retrotransposon protein Ty1-copia subclass) and C1069 (transposon protein, mutator subclass) in combination with the restriction enzymes HpaII and MspI. The methylation insensitive enzymes EcoRI and HindIII were included as controls since they generate markers completely linked to apospory when clone $\mathrm{C} 1069$ is used as a probe (Martínez et al., 2003). Analyses were initially done on DNA from the sexual (Q4188) and apomictic (Q4117) parents of the mapping cross. Hybridization with PnMAM3 showed a smeared pattern with some individual bands for the four enzymes tested. Polymorphisms between genotypes were detected on samples digested with both EcoRI and HindIII indicating genetic differences among genotypes at the specific loci. Hybridization of samples digested with HpaII and $M s p I$ revealed differences between enzymes in both plants, indicating the existence of cytosine methylation, although a similar pattern was observed for the sexual and apomictic genotypes (Figure S3). Assays with probe C1069 showed only discrete fragments. Samples digested with EcoRI and HindIII produced the expected polymorphic markers associated with apospory reported by Martínez et al. (2003) (not shown).

The hybridization patterns of samples digested with HpaII and MspI confirmed methylation of the target sequence and also revealed differences between sexual and apomictic genotypes (Figure 3A). Genotype Q4188 showed three methylation-sensitive hybridization fragments (patterns 01 or 10$)$; one of these $(\sim 0.9 \mathrm{~kb})$ was specific for the sexual plant while the other two $(\sim 3.5 \mathrm{~kb}$ and $0.5 \mathrm{~kb}$ ) were also present in Q4117 (Figure 3A). Genotype Q4117 showed two methylation-insensitive hybridization bands (pattern 11) of $\sim 2.0 \mathrm{~kb}$ and $\sim 0.7 \mathrm{~kb}$, respectively, that were absent in Q4188. Hybridization of probe C1069 on DNA from sexual (BS) and apomictic (BA) bulks showed that the three methylation-sensitive markers were monomorphic between groups. The corresponding de-bulked
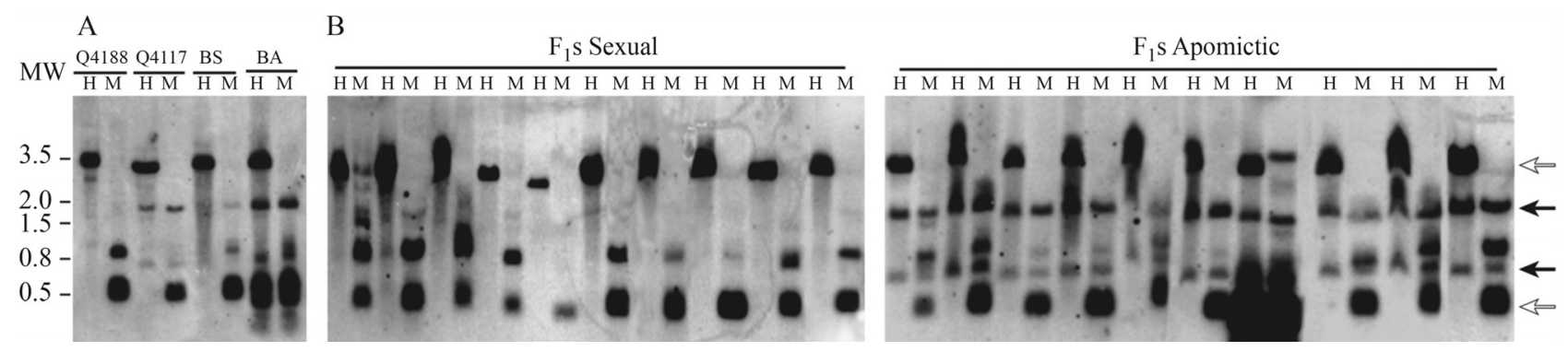

Figure 3 - Methylation-sensitive RFLP experiments with apospory-specific clone C1069. A: Hybridization done with DNA from parental genotypes Q4188 and Q4117 and sexual (BS) and apomictic (BA) bulks after digestion with HpaII (H) and MspI (M) restriction enzymes. B: Hybridization in ten sexual $\left(\mathrm{F}_{1} \mathrm{~s}\right)$ and ten aposporous $\left(\mathrm{F}_{1} \mathrm{a}\right) \mathrm{F}_{1}$ hybrids digested with $\operatorname{HpaII}(\mathrm{H})$ and $M s p \mathrm{I}(\mathrm{M})$. White arrows show methylation-sensitive markers and black arrows show methylation-insensitive markers. 
analysis confirmed that methylation-sensitive markers showed similar patterns in sexual and aposporous $\mathrm{F}_{1}$ hybrids such that no association with the reproductive mode could be detected. On the other hand, methylation-insensitive markers were specific for aposporous plants and therefore linked to apospory (Figure 3B). These polymorphisms could be attributed to: 1) an absence of the restriction site in Q4188 because of genetic variation between genotypes or 2) full methylation of the CCGG sequence. These two possibilities could not be distinguished by the approach used here.

\section{Discussion}

The availability of experimentally-generated tetraploid sexual genotypes of $P$. notatum that can be used as female parents in crosses with natural apomictic tetraploid plants has allowed the generation of populations that segregate according to the mode of reproduction without the need to use inter-specific or inter-ploid crosses. This type of segregating population has revealed some of the genetic and molecular features of apomictic reproduction and the extensive genetic variability for traits of agronomic importance in this species (Acuña et al., 2009).

The chromosome segment coding for apospory in $P$. notatum resembles the apospory-specific genomic region (ASGR) found in other grasses such as Pennisetum squamulantum (Ozias-Akins et al., 1998; Roche et al., 2001), Cenchrus ciliaris (Ozias-Akins et al., 2003; Roche et al., 1999), Paspalum simplex (Labombarda et al., 2002; Pupilli et al., 2004) and Panicum maximum (Ebina et al., 2005). In all of these species the ASGR is characterized by a lack of recombination. In Pennisetum, Cenchrus and Paspalum, there is also a strong distortion of segregation (Ozias-Akins et al., 1998, 2003; Roche et al., 1999, 2001; Labombarda et al., 2002; Pupilli et al., 2004) that is absent in P. maximum (Ebina et al., 2005). Deciphering the genetic structure of these complex non-recombinant chromosome blocks would provide key information about genes governing apospory. In P. squamulatum (Akiyama et al., 2004), C. ciliaris (Conner et al., 2008) and P. simplex (Calderini et al., 2006, 2010) this task has been attempted by sequencing BAC clones carrying molecular markers completely linked to apospory. In these three species, putative protein-coding regions as well as a large number of highly repetitive sequences have been identified (Akiyama et al., 2004; Calderini et al., 2006, 2008). Recently, several ovule transcripts originating from the ASGR-carrier chromosome were identified in Pennisetum by using a novel approach based on the comparison of two transcriptomes derived from microdissected ovules (Zeng et al., 2011).

In the present work, we used a previously classified mapping population that segregated according to mode of reproduction and a group of molecular markers linked to apospory in order to characterize sequences present in the ASGR of $P$. notatum and identify possible candidate genes.
Southern blot experiments confirmed the presence of low and high copy-number sequences in the ASGR. Hybridization bands were detected in sexual (Q4188) and apomictic (Q4117) parents of all the clones tested, indicating that the target sequences were present in both genotypes. Likewise, all contigs derived from extension of the apospory-specific marker PnMAI3 were amplified from both parental plants, with no differentiation between maternal- and paternal-specific sequences. However, since only a small part ( $\sim 5 \mathrm{~kb}$ ) of the large ASGR (estimated size: $36 \mathrm{Mbp}$ ) was characterized, hemizygosity in other regions cannot be discarded.

Only two sequences that originated from RAPD and AFLP markers aligned with cDNA clones encoding a hypothetical protein (PnMA243) and a retrotransposon element of the Ty1-copia subclass (PnMAM3), respectively. The remaining AFLP-derived sequences showed no homologies in BLAST searches, probably because of the short length of the fragments analyzed and the fact that AFLP markers often target centromeric or non-coding regions (Castiglioni et al., 1999). Rice cDNA RFLP clones mapping at the apospory locus encoded for ribosomal proteins (C996 and C569), a peptidyl-prolyl cis-trans-isomerase protein (PPIase) (C932), a KH domain containing protein (C454) and a putative mutator sub-class transposon protein (C1069). Among all these candidates, peptidyl-prolyl cis-trans isomerases (PPIases) deserve particular consideration because of their possible involvement in developmental processes (Dobson, 2004; Shaw, 2007). PPIases of the FKBP type are associated with cell division and cell elongation mediated by cytokinins and brassinosteroids in Arabidopsis (Harrar et al., 2001). Other interesting sequence corresponded to $\mathrm{KH}$ domain-containing proteins, which are RNA-binding proteins involved in mRNA stability and gene expression regulation at the posttranscriptional level (Burd and Dreyfuss, 1994; Lorkovic and Barta, 2002). In maize, $\mathrm{KH}$ proteins have been associated with the maintenance of an inactive chromatin state in knox genes within the peripheral zone of the shoot apical meristem required for proper leaf development (Buckner et al., 2008).

The extension of clone PnMAI3 by chromosome walking allowed the assembly of four contigs. PnGSA3, which mapped at the apospory-syntenic region of rice and maize, aligned with a cDNA clone of maize expressed in the endosperm and was similar to an N6-adenosinemethyltransferase MT-A70-like protein. MT-A70 proteins are mRNA methyltransferases associated with dividing tissues, particularly reproductive organs, shoot meristems and emerging lateral roots (Clancy et al., 2002; Zhong et al., 2008). Inactivation of the Arabidopsis ortholog of yeast and human mRNA adenosine methylase (MT-A) results in failure of the developing embryo to progress to the globular stage (Zhong et al., 2008). Further investigations aimed at isolating the complete sequences of the candidate genes from sexual and apomictic genotypes, and the detailed 
analysis of their expression in reproductive tissues by in situ hybridization and qRT-PCR should be done to determine possible functional associations between them and the mode of reproduction.

An in silico mapping analysis of $P$. notatum apospory-specific sequences in the rice and maize genomes identified several orthologous sequences in segments of rice chromosomes 2 and 12, previously associated with apospory in this species (Pupilli et al., 2004), and maize chromosomes 3 and 5. A recent study based on a comparative RFLP mapping strategy showed that several markers of rice chromosome 12 (including C996 and C1069 analyzed here) bracketed the chromosomal region responsible for apomixis in four Paspalum species of two taxonomic groups (Hojsgaard et al., 2011). Interestingly, the range of the ASGR estimated for $P$. squamulatum, C. ciliaris and $P$. notatum (Akiyama et al., 2004, 2005; Stein et al., 2007) agreed with the physical distances covered by aposporyspecific markers in rice chromosomes 2 and 12 and maize chromosomes 3 and 5. Considering that apomixis may have arisen from the deregulation of genes involved in sexual reproduction, the identification of coding sequences within the ASGR syntenic regions and a comparison with information derived from expression analyses could help to identify genes physically and functionally related to the trait. In another study, several transcripts differentially expressed in reproductive tissues of sexual and aposporous $P$. notatum were found to map in the same region of rice chromosome 2 (Laspina et al., 2008). Moreover, chromosome 2 of rice and chromosome 5 of maize are associated with apospory in Brachiaria hybrids (Pessino et al., 1997, 1998). Accordingly, these chromosomal segments may contain apospory-related sequences.

Several $P$. notatum apospory-specific sequences appeared to be related to repetitive elements such as mutator sub-class transposons and Ty1-copia and Ty3-gypsy subclass retrotransposons. The presence of these elements agrees with the occurrence of repetitive sequences in the ASGR of other grass species (Akiyama et al., 2004; Calderini et al., 2006; Conner et al., 2008). Moreover, nullmutants defective for the expression of a PAZ-PIWI AGO9 protein involved in the processing of transcribed retrotransposons were reported to produce non-reduced gametes in Arabidopsis thaliana, thereby mimicking the first step of aposporous development (Olmedo-Monfil et al., 2010). In a recent study of $P$. notatum, retrotransposon elements carrying transduplicated gene segments were shown to be differentially expressed in inflorescences of sexual and apomictic genotypes and a possible regulatory function of these elements in gene expression was proposed (Ochogavía et al., 2011).

Methylation-sensitive RFLP experiments indicated that, in leaves, repetitive elements located at the apospory locus were methylated, but there was no difference in the methylation pattern of apomictic and sexual genotypes.
However, Rodriguez et al. (2012) reported variation in the methylation patterns of diploid (sexual) and tetraploid (sexual and apomicts) races of $P$. notatum based on MSAP (methylation-sensitive amplification polymorphism) markers. Moreover, the inactivation of a DNA methylation pathway in maize reproductive organs resulted in apomixis-like phenotypes (Garcia-Aguilar et al., 2010). Since DNA methylation is involved in numerous biological processes, including embryogenesis, genomic imprinting, silencing of transposable elements and regulation of gene transcription (Zilberman et al., 2007) further experiments should be done on DNA extracted from inflorescences in order to better characterize the cytosine methylation of these apospory-related sequences.

\section{Acknowledgments}

The authors thank Florencia Galdeano for technical assistance. This study was financed by ANPCyT (Agencia Nacional de Promoción Científica y Tecnológica; PICT 2007 No. 00476 and PME 2006 No. 03083), Argentina, CONICET (Consejo Nacional de Investigaciones Científicas y Técnicas, PIP 112-200801-01378), Argentina. M.P., M.P.R. and L.A.S. were supported by fellowships from CONICET. S.F., E.J.M., C.L.Q., S.C.P. and J.P.A.O. are Career Members of CONICET.

\section{References}

Acuña CA, Blount AR, Quesenberry KH, Kenworthy KE and Hanna WW (2009) Bahiagrass tetraploid germplasm: Reproductive and agronomic characterization of segregating progeny. Crop Sci 49:581-588.

Akiyama Y, Conner JA, Goel S, Morishige DT, Mullet JE, Hanna WW and Ozias-Akins P (2004) High resolution physical mapping in Pennisetum squamulatum reveals extensive chromosomal heteromorphism of genomic region associated with apomixis. Plant Physiol 134:1733-1741.

Akiyama Y, Hanna WW and Ozias-Akins P (2005) High-resolution physical mapping reveals that the apospory-specific genomic region (ASGR) in Cenchrus ciliaris is located on a heterochromatic and hemizygous region of a single chromosome. Theor Appl Genet 111:1042-1051.

Altschul SF, Gish W, Miller W, Myers EW and Lipman DJ (1990) Basic local alignment search tool. J Mol Biol 215:403-410.

Asker SE and Jerling L (1992) Apomixis in Plants. CRC Press, Boca Raton, 320 pp.

Buckner B, Swaggart KA, Wong CC, Smith HA, Aurnad KM, Scanlon MJ, Schnable PS and Janic-Buckner D (2008) Expression and nucleotide diversity of the maize RIK gene. J Hered 99:407-416.

Burd CG and Dreyfuss G (1994) Conserved structures and diversity of functions of RNA-binding proteins. Science 265:615-621.

Calderini O, Chang SB, de Jong H, Busti A, Paolocci F, Arcioni S, de Vries S, Abma-Henkens MHC, Klein Lankhorst RM, Donnison IS, et al. (2006) Molecular cytogenetics and DNA sequence analysis of an apomixis linked BAC in Paspalum simplex reveal a non pericentromere location and partial 
microcolinearity with rice. Theor Appl Genet 112:11791191.

Calderini O, Donnison I, Polegri L, Panara F, Thomas A, Arcioni $\mathrm{S}$ and Pupilli F (2010) Partial isolation of the genomic region linked with apomixis in Paspalum simplex. Mol Breed 28:265-276.

Carman JG (1997) Asynchronous expression of duplicate genes in angiosperms may cause apomixis, bispory, tetraspory, and polyembryony. Biol J Linn Soc 61:51-94.

Castiglioni P, Ajmone-Marsan P, van Wijk R and Motto M (1999) AFLP markers in a molecular linkage map of maize: Codominant scoring and linkage group distribution. Theor Appl Genet 99:425-431.

Clancy MJ, Shambaugh ME, Timpte CS and Bokar JA (2002) Induction of sporulation in Saccharomyces cerevisiae leads to the formation of N6-methyladenosine in mRNA: A potential mechanism for the activity of the i.e. gene. Nucleic Acids Res 30:4509-4518.

Conner JA, Goel S, Gunawan G, Cordonnier-Pratt MM, Johnson VE, Liang C, Wang H, Pratt LH, Mullet JE, DeBarry J, et al. (2008) Sequence analysis of bacterial artificial chromosome clones from the apospory-specific genomic region of Pennisetum and Cenchrus. Plant Physiol 147:1396-13411.

Dobson CM (2004) Principles of protein folding, misfolding and aggregation. Semin Cell Dev Biol 15:3-16.

Ebina M, Nakagawa H, Yamamoto T, Araya H, Tsuruta S, Takahra M and Nakajima K (2005). Co-segregation of AFLP and RAPD markers to apospory in Guineagrass (Panicum maximum Jacq.). Grassland Sci 51:71-78.

Garcia-Aguilar M, Michaud C, Leblanc O and Grimanelli D (2010) Inactivation of a DNA methylation pathway in maize reproductive organs results in apomixis-like phenotypes. Plant Cell 22:3249-3267.

Gates RN, Quarin CL and Pedreira CGS (2004) Bahiagrass. In: Moser LE, Burson BL and Sollenberger LE (eds) WarmSeason (C4) Grasses. ASA, CSSA, and SSSA, Madison, pp 651-680.

Harrar Y, Bellini C and Faure JD (2001) FKBPs: At the crossroads of folding and transduction. Trends Plant Sci 6:426431.

Hojsgaard DH, Martínez EJ, Acuña CA, Quarin CL and Pupilli F (2011) A molecular map of the apomixis-control locus in Paspalum procurrens and its comparative analysis with other species of Paspalum. Theor Appl Genet 123:959-971.

Koltunov AM (1993) Apomixis: Embryo sacs and embryos formed without meiosis or fertilization in ovules. Plant Cell 5:1425-1437.

Labombarda P, Busti A, Caceres ME, Pupilli F and Arcioni S (2002) An AFLP marker tightly linked to apomixis reveals hemizygosity in a portion of the apomixis-controlling locus in Paspalum simplex. Genome 45:513-519.

Laspina NV, Vega T, Seijo JG, González AM, Martelotto MG, Stein J, Podio M, Ortiz JPA, Echenique V, Quarin CL, et al. (2008) Gene expression analysis at the onset of aposporous apomixis in Paspalum notatum. Plant Mol Biol 67:615-628.

Lorkovic ZJ and Barta A (2002) Genome analysis: RNA recognition motif (RRM) and $\mathrm{K}$ homology $(\mathrm{KH})$ domain RNAbinding proteins from the flowering plant Arabidopsis thaliana. Nucleic Acids Res 30:623-635.
Martínez EJ, Urbani MH, Quarin CL and Ortiz JPA (2001) Inheritance of apospory in bahiagrass, Paspalum notatum. Hereditas 135:19-25.

Martínez EJ, Hopp E, Stein J, Ortiz JPA and Quarin CL (2003) Genetic characterization of apospory in tetraploid Paspalum notatum based on the identification of linked molecular markers. Mol Breed 12:319-327.

McClelland M, Nelson M and Raschke E (1994) Effect of sitespecific modification on restriction endonucleases and DNA modification methyltransferases. Nucleic Acids Res 22:3640-3659.

Michelmore RW, Paran I and Kesselli V (1991) Identification of markers linked to disease-resistance genes by bulked segregant analysis: A rapid method to detect markers in specific genomic regions by using segregating populations. Proc Natl Acad Sci USA 88:9828-9832.

Nogler GA (1984) Gametophytic apomixis. In: Johri BM (ed) Embryology of Angiosperms. Springer-Verlag, Berlin, pp 475-518.

Ochogavía AC, Seijo JG, González MA, Podio M, Silveira ED, Machado Lacerda AL, Carneiro VTC, Ortiz JPA and Pessino SC (2011) Characterization of retrotransposon sequences expressed in inflorescences of apomictic and sexual Paspalum notatum plants. Sexual Plant Reprod 24:231-246.

Olmedo-Monfil V, Durán-Figueroa N, Arteaga-Vázquez M, Demesa-Arévalo E, Autran D, Grimanelli D, Slotkin RK, Martienssen RA and Vielle-Calzada JP (2010) Control of female gamete formation by a small RNA pathway in Arabidopsis. Nature 464:628-632.

Ortiz JPA, Pessino SC, Leblanc O, Hayward MD and Quarin CL (1997) Genetic fingerprinting for determining the mode of reproduction in Paspalum notatum, a subtropical apomictic forage grass. Theor Appl Genet 95:850-856.

Ortiz JPA, Pessino SC, Bhat V, Hayward MD and Quarin CL (2001) A genetic linkage map of diploid Paspalum notatum. Crop Sci 41:823-830.

Ozias-Akins P and van Dijk P (2007) Mendelian genetics of apomixis in plants. Annu Rev Genet 41:509-537.

Ozias-Akins P, Roche D and Hanna WW (1998) Tight clustering and hemizygosity of apomixis-linked molecular markers in Pennisetum squamulatum implies genetic control of apospory by divergent locus that may have no allelic form in sexual genotypes. Proc Natl Acad Sci USA 95:5127-5132.

Ozias-Akins P, Akiyama Y and Hanna WW (2003) Molecular characterization of the genomic region linked with apomixis in Pennisetum/Cenchrus. Funct Integr Genomics 3:94-104.

Pessino SC, Ortiz JPA, Leblanc O, do Valle CB, Evans C and Hayward MD (1997) Identification of a maize linkage group related to apomixis in Brachiaria. Theor Appl Genet 94:439-444.

Pessino SC, Evans C, Ortiz JPA, Armstead I, do Valle CB and Hayward MD (1998) A genetic map of the apospory-region in Brachiaria hybrids: Identification of two markers closely associated with the trait. Hereditas 128:153-158.

Pupilli F, Martínez EJ, Busti A, Calderini O, Quarin CL and Arcioni S (2004) Comparative mapping reveals partial conservation of synteny at the apomixis locus in Paspalum spp. Mol Genet Genomics 270:539-548.

Quarin CL, Urbani MH, Blount AR, Martínez EJ, Hack CM, Burton GW and Quesenberry KH (2003) Registration of Q4188 
and Q4205, sexual tetraploid germplasm lines of Bahiagrass. Crop Sci 43:745-746.

Rebozzio RN, Rodriguez MP, Stein J, Quarin CL, Ortiz JPA and Espinoza F (2012) Validation of molecular markers linked to apospory in tetraploid races of bahiagrass, Paspalum notatum Flüggé. Mol Breed 29:189-198.

Roche D, Cong P, Chen Z, Hanna WW, Gustine DL, Sherwood RT and Ozias-Akins P (1999) An apospory-specific genomic region is conserved between buffelgrass (Cenchrus ciliaris L.) and Pennisetum squamulatum Fresen. Plant J 19:203-208.

Roche D, Chen Z, Hanna WW and Ozias-Akins P (2001) NonMendelian transmission of an apospory-specific genomic region in a reciprocal cross between sexual pearl millet (Pennisetum glaucum) and an apomictic F1 (P. glaucum $\mathrm{x} P$. squamulatum). Sex Plant Reprod 13:217-223.

Rodriguez MP, Cervigni GDL, Quarin CL and Ortiz JPA (2012) Frequencies and variation in cytosine methylation patterns in diploid and tetraploid cytotypes of Paspalum notatum assessed by MSAP markers. Biol Plant 56:276-282.

Salse J, Piégu B, Cooke R and Delseny M (2004) New in silico insight into the synteny between rice (Oryza sativa L.) and maize (Zea mays L.) highlights reshuffling and indentifies new duplications in the rice genome. Plant J 38:396-409.

Savidan Y (2000) Apomixis: Genetics and breeding. In: Janick J (ed) Plant Breeding Reviews. John Wiley \& Sons, London, pp 13-86.

Shaw PE (2007) Peptidyl-prolyl cis/trans isomerases and transcription: Is there a twist in the tail? EMBO Rep 8:40-45.

Spillane C, Curtis M and Grossniklaus U (2004) Apomixis technology development - Virgin births in farmers' fields? Nat Biotechnol 22:687-691.

Stein J, Quarin CL, Martínez EJ, Pessino SC and Ortiz JPA (2004) Tetraploid races of Paspalum notatum show polysomic inheritance and preferential chromosome pairing around the apospory-controlling locus. Theor Appl Genet 109:186-191.

Stein J, Pessino SC, Martínez EJ, Rodríguez MP, Siena L, Quarin CL and Ortiz JPA (2007) A genetic map of tetraploid Paspalum notatum Flugge (bahiagrass) based on singledose molecular markers. Mol Breed 20:153-166.

Tardy-Planechaud S, Fujimoto J, Lin SS and Sowers LC (1997) Solid phase synthesis and restriction endonuclease cleavage of oligodeoxynucleotides containing 5-(hydroxymethyl)-cytosine. Nucleic Acids Res 25:553-558.

Toenniessen GH (2001) Feeding the world in the 21st century: Plant breeding, biotechnology, and the potential role of apomixis. In: Savidan Y, Carman JG and Dresselhaus T (eds) The Flowering of Apomixis: From Mechanisms to Genetic Engineering. CIMMYT, Mexico, D.F., pp 1-7.
Vielle-Calzada JP, Crane CF and Stelly DM (1996) Apomixis: The asexual revolution. Science 274:1322-1323.

Zeng Y, Conner J and Ozias-Akins P (2011) Identification of ovule transcripts from the apospory-specific genomic region (ASGR)-carrier chromosome. BMC Genomics 12:206.

Zilberman D, Gehring M, Tran RK, Ballinger T and Henikoff S (2007) Genome-wide analysis of Arabidopsis thaliana DNA methylation uncovers an interdependence between methylation and transcription. Nat Genet 39:61-69.

Zhong S, Li H, Bodi Z, Button J, Vespa L, Herzog M and Fray R (2008) MTA is an Arabidopsis messenger RNA adenosine methylase and interacts with a homolog of a sex-specific splicing factor. Plant Cell 20:1278-1288.

\section{Internet Resources}

Rice Genome Research Program, Japan, http://rgp.dna.affrc.go.jp/E/Publicdata.html (November 15, 2011).

GRAMENE, http://www.gramene.org (November 15, 2011).

MaizeSequence, http://www.maizesequence.org (November 15, 2011).

TIGR Rice Genome Annotation, http://blast.jcvi.org/eukblast/index.cgi?project=osa1 (November 15,2011$)$.

\section{Supplementary Material}

The following online material is available for this article:

Table S1 - Primers used for extending PnMAI3 marker.

Table S2 - Primers for PnMAI3 contigs amplification from genomic DNA.

Figure S1 - Amplification of contigs PnGSA1-4 from genomic DNA.

Figure S2 - Mapping PnGSA1 in Paspalum notatum.

Figure S3 - Methylation-sensitive RFLP analysis carried out with clone PnMAM3.

This material is available as part of the online article from http://www.scielo.br/gmb.

Associate Editor: Marcia Pinheiro Margis

License information: This is an open-access article distributed under the terms of the Creative Commons Attribution License, which permits unrestricted use, distribution, and reproduction in any medium, provided the original work is properly cited. 
Table S1 - Primers used to extend the apospory-specific AFLP-derived sequence PnMAI3.

\begin{tabular}{|c|c|}
\hline Primer name & Sequence \\
\hline $\mathrm{I} 3 \mathrm{NP} 31^{1}$ & AAATTCGGCTTCACGGCATTGGTCATT \\
\hline $\mathrm{I}^{3} \mathrm{NP} 32^{2}$ & GATGCCTTGGGTCTTCTCTTATACGTT \\
\hline I3NP5 $^{1}$ & CAGGCGTCGGACGTTCCAGTGAATGCA \\
\hline $\mathrm{I} \mathrm{NP} 52^{2}$ & CTAGGGGTCCGATTGGTTGCTTGAATT \\
\hline
\end{tabular}


Table S2 - Primers used to amplify contigs derived from extension of the apospory-specific sequence PnMAI3 from Paspalum notatum genomic DNA.

\begin{tabular}{|c|c|c|c|}
\hline PnMAI3 anchor primer $\left(5^{\prime}-3^{\prime}\right)$ & $\begin{array}{l}\text { Contig } \\
\text { sequence }\end{array}$ & Specific-primer of the extended sequence $\left(5^{\prime}-3^{\prime}\right)$ & $\begin{array}{l}\text { Length } \\
\text { (bp) }\end{array}$ \\
\hline VI3R: CAATGACCAATGCCGTGAAGC & $\begin{array}{l}\text { PnGSA1 } \\
\text { PnGSA2 }\end{array}$ & $\begin{array}{l}\text { VI35BF1: GGCTGGTATCTAGCAGCTCAA } \\
\text { VI35BF2: GCATGTTCAAGCACATCTATC } \\
\text { VI354BF2: CAGAAGGCCAGAAGAACTCA }\end{array}$ & $\begin{array}{l}309 \\
279 \\
291\end{array}$ \\
\hline VI3F: CCCAGACCGTTCGATATGTGTAAT & $\begin{array}{l}\text { PSGSA3 } \\
\text { PnGSA4 }\end{array}$ & $\begin{array}{l}\text { VI338AR1: GGAATCCATCCTGAAGTTTCG } \\
\text { VI338AR2: GCTCGTAGTGGACATATTTGC } \\
\text { VI3310AR1: TAGTCATTGGCGGTGGTGGAT } \\
\text { VI3310AR2: GTGAGTGCCAGGAACTCTTCT }\end{array}$ & $\begin{array}{l}538 \\
417 \\
342 \\
774\end{array}$ \\
\hline
\end{tabular}


Figure S1: PCR amplification of contigs PnGSA1-4 from genomic DNA

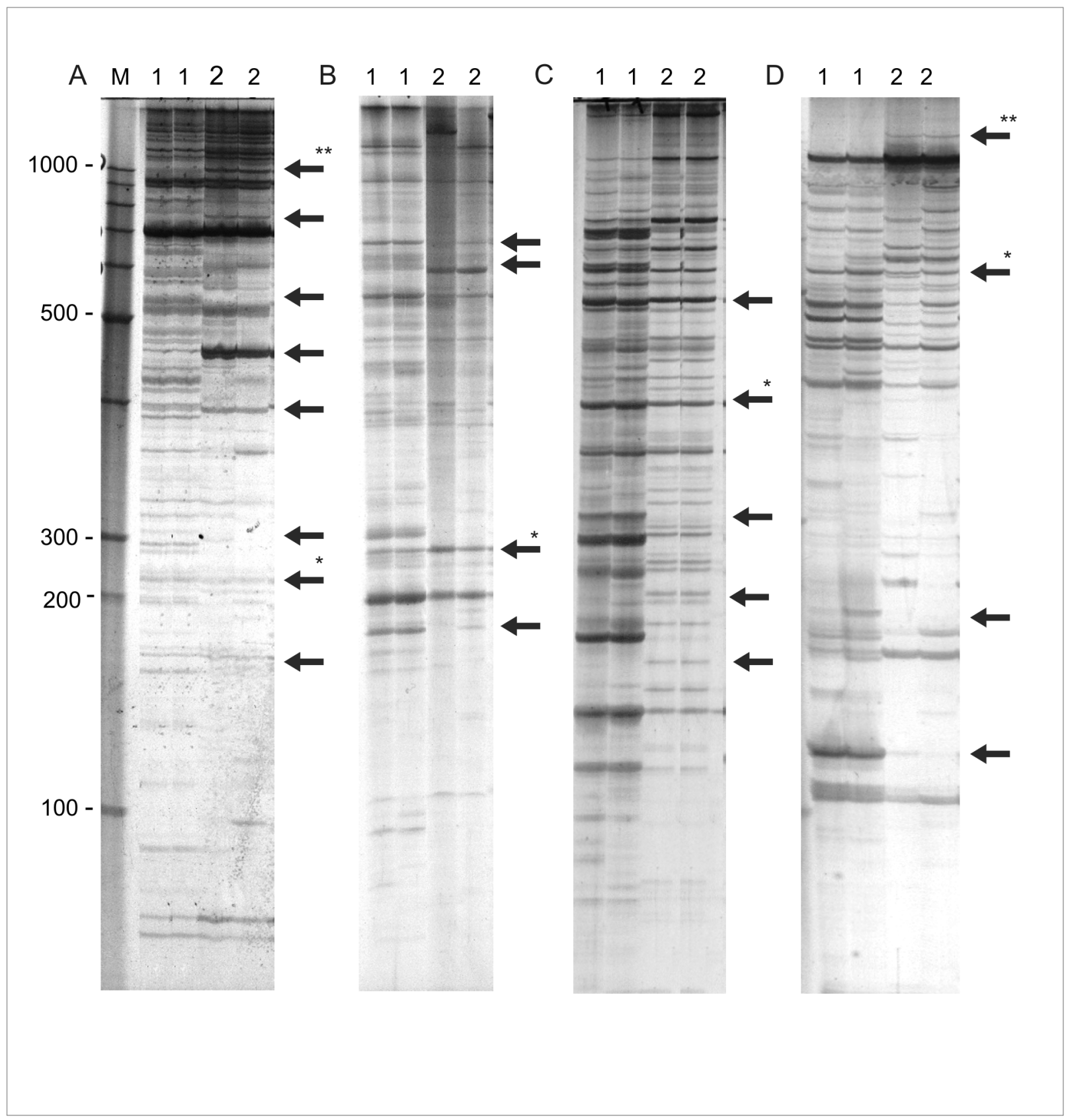

A, B, C and D: Acrylamide gels showing amplification products derived from PnGSAl (with primers VI3R - VI35BF1), PnGSA2 (with primers VI3R - VI354BF2), PnGSA3 (VI3FVI338AR1) and PnGSA4 (with primers VI3F- VI3310AR2) (Table S2). M: molecular weight marker. Arrows indicated polymorphic fragments between parental genotypes. *indicates fragments of the expected size according to the original sequence. ** indicate markers linked to apospory. 
Figure S2: PCR amplification of contig PnGSA1 in a sample of Paspalum notatum mapping population

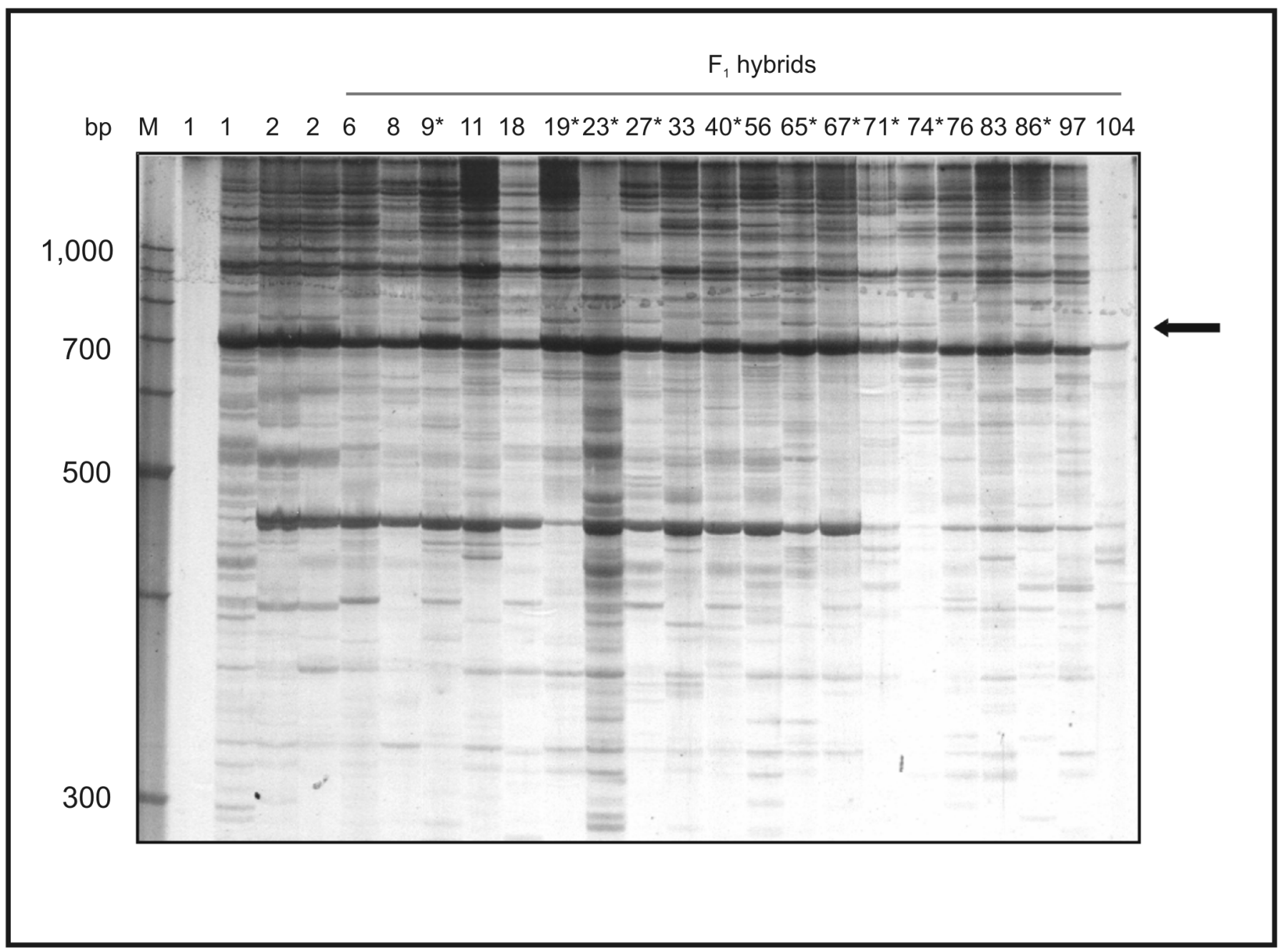

Acrylamide gels showing amplification products derived from the PnGSA1 with primers VI3R and VI35BF1 (Table S2). M: molecular weight marker. 1 and 2: DNA samples from Q4188 and Q4117 loaded by duplicate (the amplification in the first lane of Q4188 failed) . 6-104: experimental number of $\mathrm{F}_{1}$ hybrids of the mapping population. *aposporous plants. Arrow indicated the band. cosegregating with apospory. 
